\title{
FE Simulation of the Stress-Strain State during Shear-Compression Testing and Asymmetric Three-Roll Rolling Process
}

\author{
Alexander Pesin, Denis Pustovoytov, Veniamin Kharitonov and Aleksey Korchunov \\ Nosov Magnitogorsk State Technical University, 455000, Magnitogorsk, Lenin prospect, 38, Russia
}

\begin{abstract}
A three-roll rolling process is a significant technique in the production of wire rod, round bars and hexagonal profiles for structural applications. Better mechanical properties of wire rod, round bars and hexagonal profiles can be achieved due to large plastic deformation by the three-roll rolling process. Asymmetric rolling is a novel technique characterized by a kinematic asymmetry linked to the difference in peripheral speed of the rolls, able to introduce additional shear strains through the bar thickness. Physical simulation of shear strain, which is similar to that occurring in asymmetric three-roll rolling process, is very important for design of technology of producing ultrafine grain materials. Shear testing is complicated by the fact that a state of large shear is not easily achievable in most specimen geometries. Application of the shear-compression testing and specimen geometry to physical simulation of asymmetric three-roll rolling process is discussed in the paper. FEM simulation and comparison of the stress-strain state during shear-compression testing and asymmetric three-roll rolling process is presented. The results of investigation can be used to optimize the physical simulation of asymmetric three-roll rolling processes and for design of technology of producing ultrafine grain materials by severe plastic deformation.
\end{abstract}

\section{Introduction}

A three-roll rolling process is a significant technique in the production of wire rod, round bars and hexagonal profiles for structural applications. It is a rolling process in which material is shaped by three rolls in three directions, everyone of which is inclined to the others at $120^{\circ}$ [1], [2]. Three-roll rolling process has various advantages such as easy flow control, small space requirement, and comparatively uniform deformation, etc. The restriction of the material imposed by the shape of caliber rolls in the three-roll rolling process is greater than that in the two-roll rolling process. The accuracy of the shape of products made by the three-roll rolling process is considered to be higher than that of the tworoll rolling process [3].

It is well known that efficient way to increase strength of metallic materials is to form an ultra-fine grain structure using severe plastic deformation (SPD) methods [4]. Nowadays many variants of SPD techniques are available. Asymmetric rolling process is one of them [5][7]. Contrary to other SPD methods such as high pressure torsion and equal channel angular pressing, the asymmetric rolling allows to provide the possibility for overcoming the limitation of producing UFG materials with large dimensions due to its continuous feature [8][9]. Among the asymmetric rolling processes, the differential speed rolling in which the speeds of the rolls are different, is considered to be the most effective for achieving UFG structure of materials [10]-[13]. Better mechanical properties of wire rod, round bars and hexagonal profiles can be achieved due to large plastic deformation in asymmetric three-roll rolling process. symmetric rolling results in the development of strain with strong shear components, both at the surfaces and at the center of the workpiece.

Physical simulation of shear and compression strains, which are similar to that occurring in asymmetric threeroll rolling processes, is very important for design of technology because it allows to consider a variety of technological variants and to find the best solution in relatively short time and with low costs. Shear testing is complicated by the fact that a state of large shear is not easily achievable in most specimen geometries. Problem of selection of shear testing and specimen geometry has been discussed in numerous publications.

A shear-tensile specimen was developed by Hundy and Green [14]. Another example of such a specimen is the so-called "hat specimen" which was developed by Meyer and Manwaring [15], and used thereafter by many other researchers. Another variant is the double shear specimen used by Klepaczko [16]. Very simple specimen geometry (thin foil) has been used by Clifton and Klopp [17] in their pressure-shear plate impact experiments. Peirs, et al. [18] introduced the eccentric notch shear specimen, which was designed for shear testing of sheet metals over a wide range of strain rates. A shearcompression specimen for large strain testing of materials was developed by Rittel et al. [19]. The specimen consists of a cylinder having an inclined gauge section 
created by two diametrically opposed rectangular slots which are machined at $45^{\circ}$ with respect to the longitudinal axis. The specimen was used in numerous investigations, including constitutive testing, texture evolution, and adiabatic shear banding. A miniature version of the shear-compression specimen was used for the characterization of nanomaterials. The modified shear-compression specimen was presented in [20]. In the modified specimen, the two diametrically machined gauges were semi-circular (instead of rectangular). This modification induces large strains on the mid-section of the gauge without the sharp edges and stress concentrations of the former rectangular gauges. Although many studies have been done, but there are no numerical data on the analysis of the applicability of the shear-compression testing to the modeling of the asymmetric three-roll rolling processes. The goal of this research is the numerical simulation and comparison of the stress-strain state of metal during shear-compression testing and asymmetric three-roll rolling process. The results of investigation can be used to optimize the physical simulation of asymmetric three-roll rolling processes and for design of technology of producing UFG materials by SPD.

\section{Research method}

DEFORM 3D, based on finite element method (FEM), was used to analyze the stress-strain state of metal during shear-compression testing and asymmetric three-roll rolling process. Simulation of the processes was performed at room temperature. Steel AISI 1010 from DEFORM 3D material library was chosen as a hardened rigid-plastic material for specimen and bar.

The specimen consisted of a parallelepiped having an inclined gauge section created by two diametrically opposed semi-circular slots which were machined at $45^{\circ}$. The level of strain can be controlled through adjustment of the dimensions and height reduction of the specimen during shear-compression testing. Height of the specimen was $24 \mathrm{~mm}$, section dimensions were $12 \times 12 \mathrm{~mm}$, gauge thickness was $1.5 \mathrm{~mm}$ and gauge width was $3 \mathrm{~mm}$. (Fig. 1). The specimen provides dominant shear strain in an inclined gauge-section. The specimen was meshed with 80325 tetrahedral elements. A Coulomb friction model was used between dies and specimen. The friction coefficient on the contact surfaces was 0.4. A vertical displacement of the top die was $2.4 \mathrm{~mm}$. It corresponded to $10 \%$ of height reduction. Velocity of displacement was $2.0 \mathrm{~mm} / \mathrm{sec}$.

The specimen was compressed between two flat dies during shear-compression testing in accordance to the scheme (Fig. 2).

Previously [12]-[13] it was found that to obtain a high uniform shear strain through the bar thickness during asymmetric three-roll rolling process is necessary: 1) very high contact friction; 2) high elongation ratio; 3) speed ratio of rolls should be equal to elongation ratio per pass. Schematic illustration of the asymmetric threeroll rolling process in a stand with two three-roll calibers located very close to each other is shown in Fig. 3.
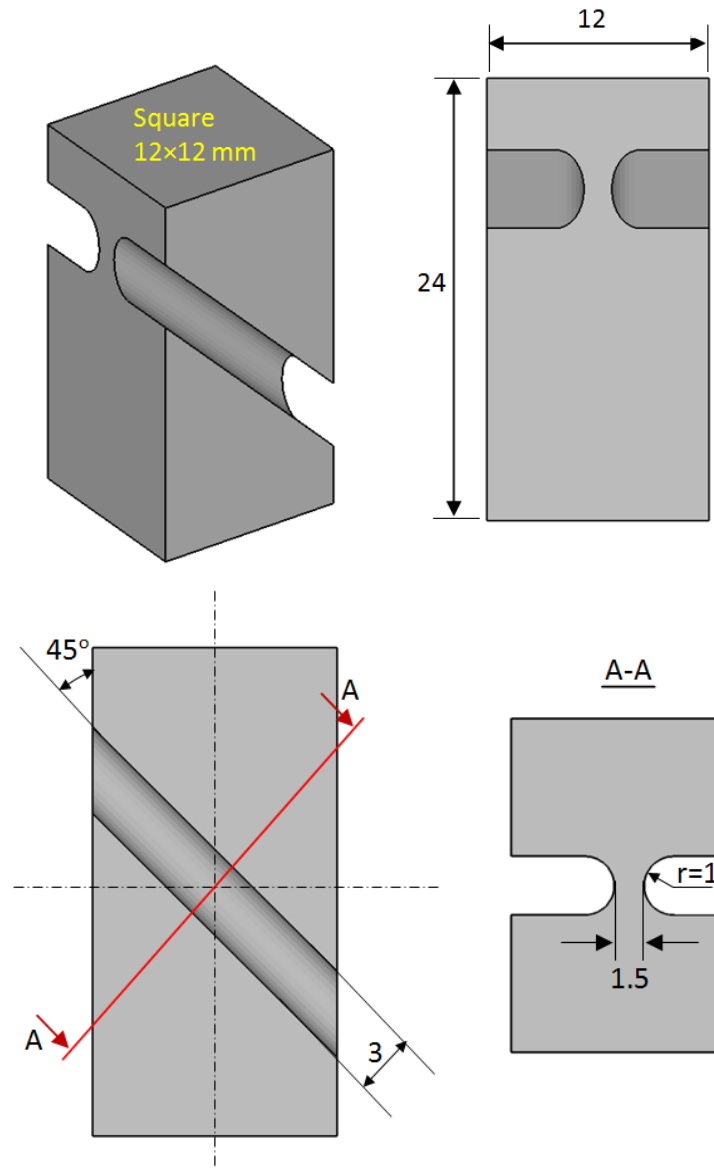

$\underline{A-A}$

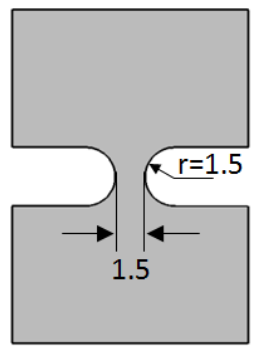

Figure 1. Geometry of the specimen.

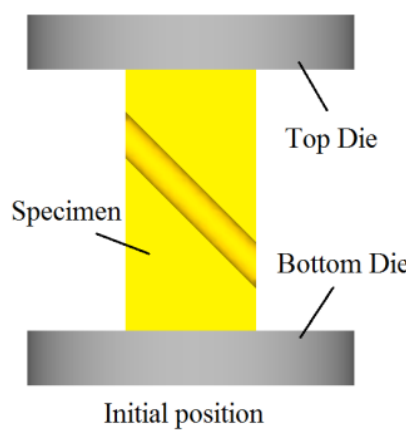

Step 1

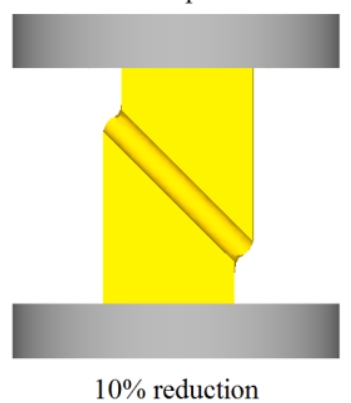

Step 2

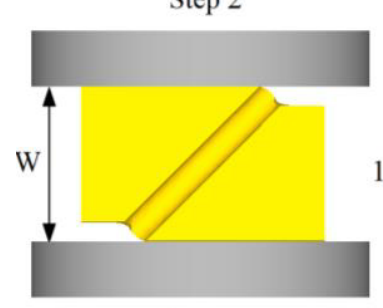

$90^{\circ}$ rotation

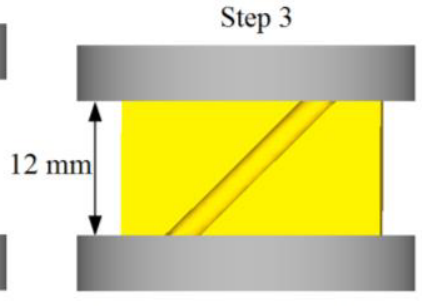

reduction from $\mathrm{W}$ to $12 \mathrm{~mm}$

Figure 2. Schematic illustration of the shear-compression testing for one cycle.

A round bar of $\mathrm{d}=4.0 \mathrm{~mm}$ diameter of AISI 1010 was used. The shape of the first caliber was triangle. The shape of the second caliber was round. The initial and the final shape of the bar was a round. The modeling of the process was performed at room temperature without taking into account the increment of the metal's 
temperature due to the thermal effect of deformation. The isothermal condition was fulfilled due to low rolling speed. The work rolls were assumed as rigid. Peripheral speeds of the rolls were set according to the relationship (1). Peripheral speed of the first roll in all calculation variants was set constant $\left(\mathrm{V}_{1}=0.2 \mathrm{~m} / \mathrm{sec}\right)$. The three-roll rolling process was carried out with total elongation ratio 2. Speed asymmetry of the work rolls was equal to elongation of the bar material.

$$
V_{2}=V_{3}=V_{5}=V_{6}=\frac{S_{0}}{S_{1}} V_{1}, V_{4}=\frac{S_{0}}{S_{2}} V_{1}
$$

where $V_{1} \ldots V_{6}$ - peripheral speeds of the rolls; $S_{0}-$ the initial cross section area of the bar; $S_{1}$ - the cross section area of the bar after the first three-roll pass; $S_{2}-$ the cross section area of the bar after the second three-roll pass.

Bar was meshed with 80630 tetrahedral elements. A Coulomb friction model was used between rolls and bar. The friction coefficient on the contact surfaces was 0.4 .
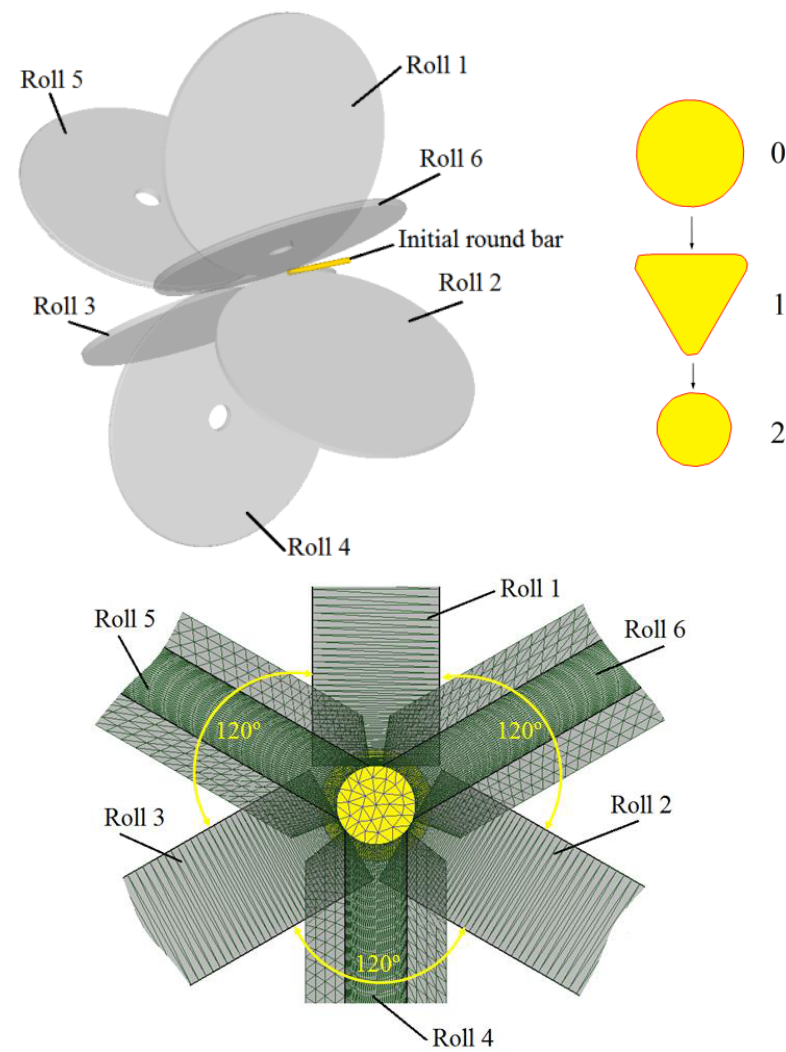

Figure 3. Schematic illustration of the asymmetric three-roll rolling process $(0$ - initial shape; 1 - after the first pass; 2 - after the second pass).

\section{Simulation results and discussion}

The deformed specimen after one cycle of the shearcompression testing is shown in Fig. 4. Strain is localized in gauge section and it is typical for large plastic deformation.

It should be noted that the dimensions of the specimen are the important factors that influence on the level of strain during shear-compression testing. With decreasing the gauge thickness and the gauge width as well as with increasing the height reduction the effective strain is increased. So the level of strain can be controlled through adjustment of the dimensions and height reduction of the specimen during shear-compression testing. The friction coefficient on the contact surfaces does not influence on the stress-strain state of metal during shear-compression testing. However the height reduction of the specimen during shear-compression testing is limited by its destruction. End effects are noticeable on both fillets of the gauge. The maximum height reduction should be established from experimental testing. The results of the stress-strain state of metal during shear-compression testing were compared with asymmetric three-roll rolling process. Distributions of the effective stress and strain along the mid-section of the gauge are shown in Fig. 4. The uniform distributions can clearly be observed during shear-compression testing of the specimen. Distributions of the effective stress and strain along the bar during asymmetric three-roll rolling process are shown in Fig. 5. It also can be observed the uniform distributions of the effective stress and strain. Effective stress is $667 \mathrm{MPa}$ and effective strain is 1.0. It quite corresponds to shearcompression testing when height reduction of the specimen is $10 \%$.
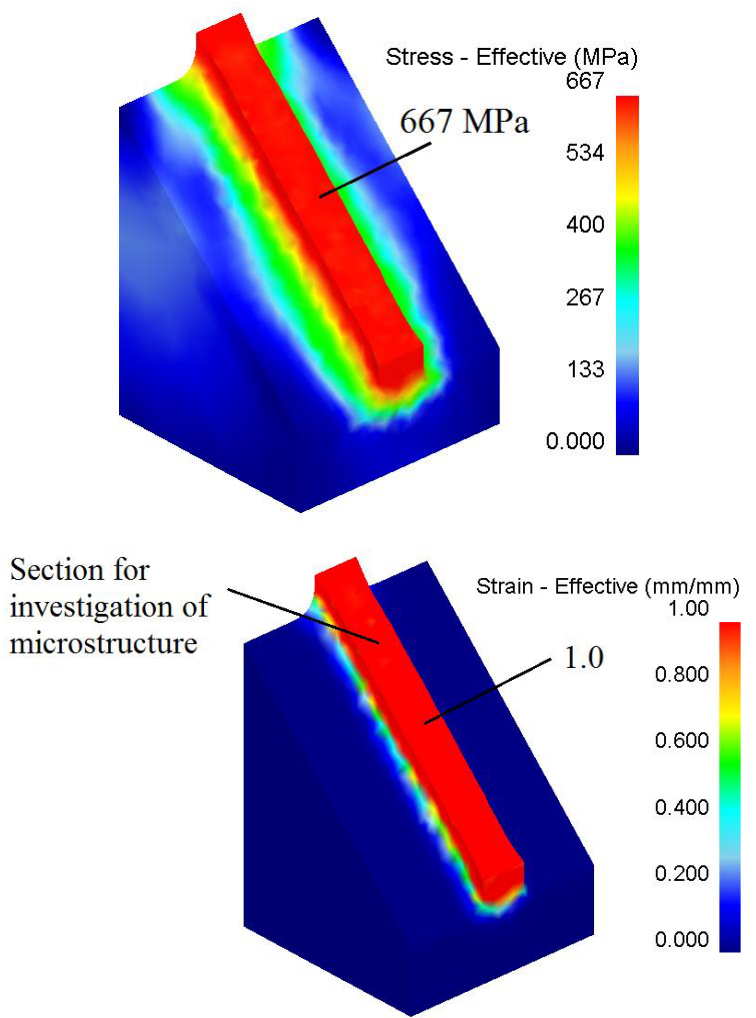

Figure 4. Fields of the effective stress and the effective strain after one cycle of the shear-compression testing.

Change in a Lagrange's mesh before and after shearcompression testing is shown in Fig. 6-Fig.7. Single cell with initial shape of square with dimensions $0.25 \times 0.25 \mathrm{~mm}$ became the parallelogram. The height of the square was reduced from $0.25 \mathrm{~mm}$ to $0.16 \mathrm{~mm}$. but the length was not elongated. Lack of elongation can be explained by high metal flow in width direction. 

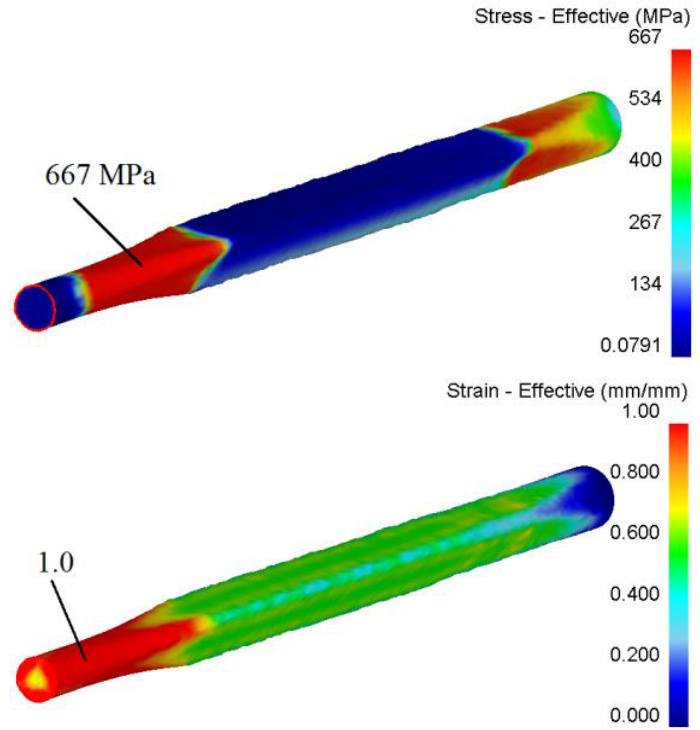

Figure 5. Fields of the effective stress and the effective strain during asymmetric three-roll rolling process with total elongation ratio 2 .

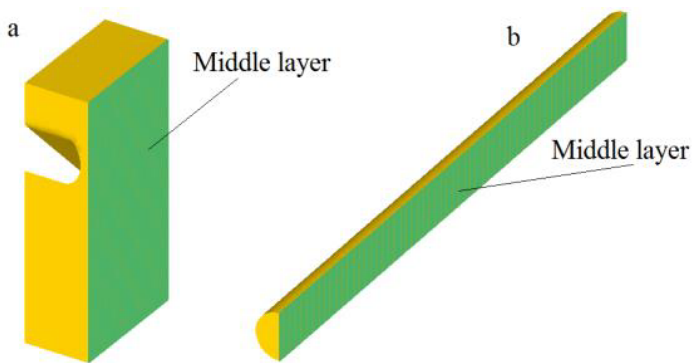

Figure 6. Position of the Lagrange's mesh: a - specimen for shear-compression testing; $b$ - bar for asymmetric three-roll rolling process.

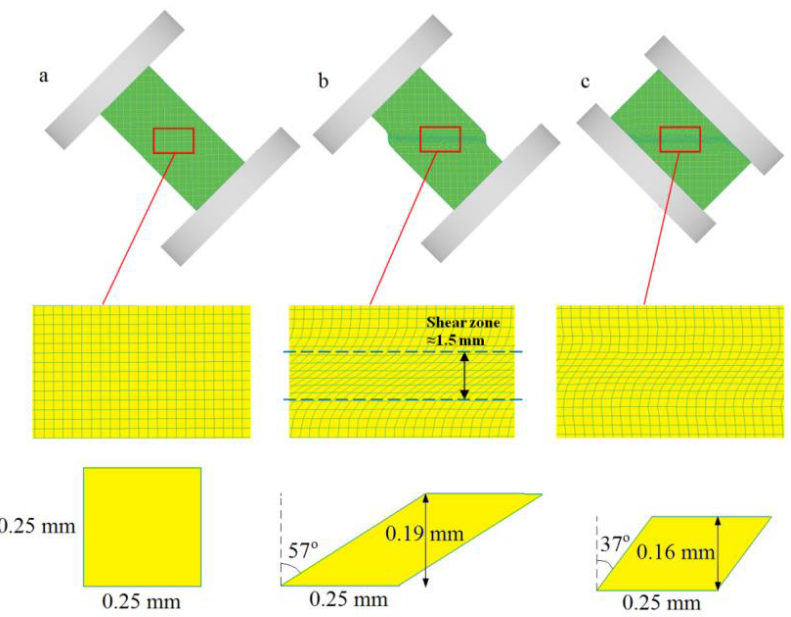

Figure 7. Change in Lagrange's mesh after one cycle of the shear-compression testing: $\mathrm{a}$ - initial mesh; $\mathrm{b}$ - mesh after step $1 ; \mathrm{c}-$ mesh after step 3

Shear angle was increased to $57^{\circ}$ after the first step and it was decreased to $37^{\circ}$ after the second step (Fig. 7). Shear strain can be presented as tangent of shear angle. The shear zone in gauge section was approximately $1.5 \mathrm{~mm}$ after $10 \%$ of height reduction. Change in a Lagrange's mesh before and after asymmetric three-roll rolling process is shown in Fig. 8. Single cell with initial shape of square with dimensions $0.25 \times 0.25 \mathrm{~mm}$ also became the parallelogram. The height of the square was reduced from $0.25 \mathrm{~mm}$ to $0.15 \mathrm{~mm}$ and the length was elongated from 0.25 to $0.50 \mathrm{~mm}$. Shear angle was increased to $39^{\circ}$ (Fig. 8). The shape of Lagrange's mesh in both cases (during shear-compression testing and asymmetric three-roll rolling process) highlights the remarkable action of the shear strain.

The accumulated strain which is equal to 1.0 can be observed during the shear-compression testing for one cycle. It corresponds to asymmetric three-roll rolling process with total elongation ratio 2 . It is well known that UFG structure can be observed when accumulated strain is about 3.0...4.0. Physical simulation of the shearcompression testing for four cycles (when accumulated strain is 4.0) was performed. It quite corresponded to asymmetric three-roll rolling process with total elongation ratio 8 . The microstructure of the specimen after four cycles of the shear-compression testing were examined with a scanning electron microscope (SEM, JSM-6490LV) at accelerating voltage of $20 \mathrm{kV}$. Section for investigation of microstructure is shown in Fig. 4. UFG structure was obtained (Fig. 9). Thereby the shearcompression testing can be applied to physical simulation of asymmetric three-roll rolling process. Further experimental investigation of microstructure of steel AISI 1010 processed by asymmetric three-roll rolling process is required.
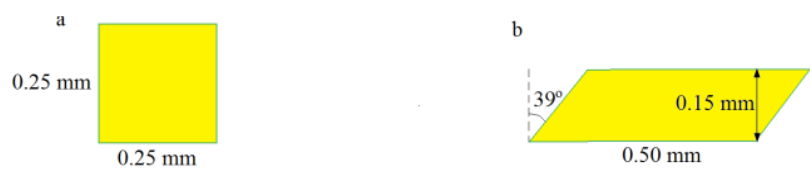

Figure 8. Change in Lagrange's mesh during asymmetric threeroll rolling process: $\mathrm{a}$ - initial mesh; $\mathrm{b}-$ mesh after the second rolling pass.

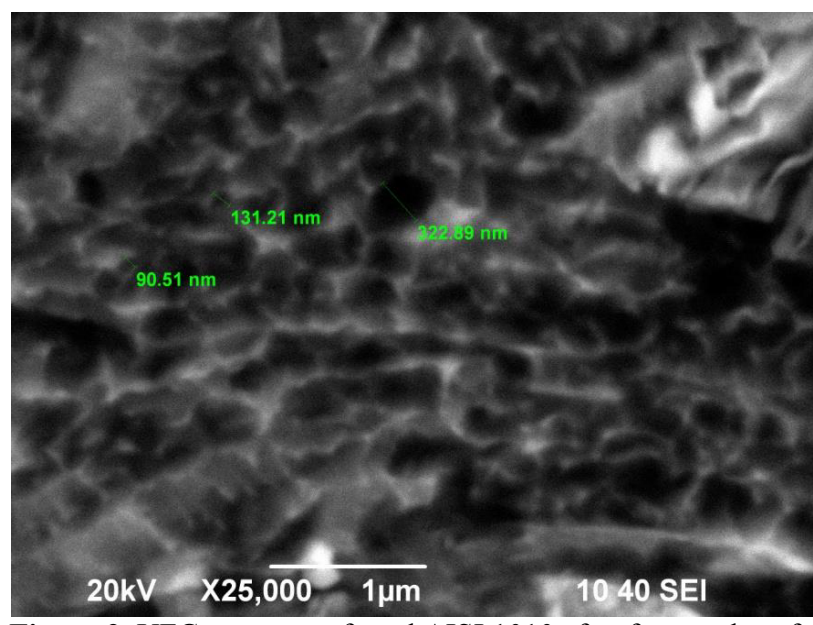

Figure 9. UFG structure of steel AISI 1010 after four cycles of the shear-compression testing (accumulated strain is 4.0 ).

\section{Conclusions}

FEM simulations and comparison of the stress-strain state of steel AISI 1010 during shear-compression testing and asymmetric three-roll rolling process were studied. The uniform distribution of the effective stress $(667 \mathrm{MPa})$ and the effective strain (1.0) can be observed during 
asymmetric three-roll rolling process in a stand with two three-roll calibers located very close to each other. The three-roll rolling process was carried out with total elongation ratio 2 . It quite corresponds to the stress-strain state of steel AISI 1010 during shear-compression testing when the specimen consists of a parallelepiped having an inclined gauge section created by two diametrically opposed semi-circular slots which are machined at $45^{\circ}$. Height of the specimen is $24 \mathrm{~mm}$, section dimensions are $12 \times 12 \mathrm{~mm}$, height reduction is $10 \%$, gauge thickness is $1.5 \mathrm{~mm}$ and gauge width is $3 \mathrm{~mm}$. Thereby the shearcompression testing can be applied to physical simulation of asymmetric three-roll rolling process. The results of investigation can be used to optimize the physical simulation of asymmetric three-roll rolling processes and for design of technology of producing ultrafine grain materials by severe plastic deformation. Further experimental investigation is required.

\section{Acknowledgement}

The research work was carried out in accordance with the government order of the Ministry of Education and Science of the Russian Federation executed by Nosov Magnitogorsk State Technical University on the topic «Development of technology for manufacture of highstrength long rolled sections from materials with ultrafine grain structure using combined processes of severe plastic deformation» (project No $11.1525 \mathrm{~K}$ from 18.07.2014).

\section{References}

1. K. Komori, Simulation of deformation and temperature in multi-pass three-roll rolling, Journal of Materials Processing Technology. 92-93, 450-457, (1999)

2. C. Overhagen, P. Mauk, A new rolling model for three-roll rolling mills, Key Engineering Materials. 622-623, 879-886, (2014)

3. J.H. Min, H.C. Kwon, Y. Lee, J.S. Woo, Y.T. Im, Analytical model for prediction of deformed shape in three-roll rolling process, Journal of Materials Processing Technology. 140, 471-477, (2003)

4. Laszlo S. Toth, Chengfan Gu, Ultrafine-grain metals by severe plastic deformation, Materials Characterization. 92, 1-14, (2014)

5. Zhiming $\mathrm{Li}$, Liming $\mathrm{Fu}$, Bin Fu, Aidang Shan, Effects of annealing on microstructure and mechanical properties of nano-grained titanium produced by combination of asymmetric and symmetric rolling, Materials Science and Engineering: A. 558, 309-318, (2012)

6. Y.H. Ji, J.J. Park, Development of severe plastic deformation by various asymmetric rolling processes,
Materials Science and Engineering: A. 499, 14-17, (2009)

7. Kyung-Moon Lee, Hu-Chul Lee, Grain refinement and mechanical properties of asymmetrically rolled low carbon steel, Journal of Materials Processing Technology. 210, 1574-1579, (2010)

8. W.J. Kim, B.G. Hwang, M.J. Lee, Y.B. Park, Effect of speed-ratio on microstructure, and mechanical properties of $\mathrm{Mg}-3 \mathrm{Al}-1 \mathrm{Zn}$ alloy, in differential speed rolling, Journal of Alloys and Compounds. 509 8510-8517, (2011)

9. K. Bobor, Z. Hegedus, J. Gubicza, I. Barkai, P. Pekker, G. Krallics, Microstructure and mechanical properties of Al 7075 alloy processed by differential speed rolling, Periodica Polytechnica Mechanical Engineering. 56, 111-115, (2012).

10. Loorentz, Y.G. Ko, Effect of differential speed rolling strain on microstructure and mechanical properties of nanostructured $5052 \mathrm{Al}$ alloy, Journal of Alloys and Compounds. 586, S205-S209, (2014).

11. Q. Cui, K. Ohori, Grain refinement of high purity aluminum by asymmetric rolling, Materials Science and Technology. 16, 1095-1101, (2000).

12. A. Pesin, M. Chukin, A. Korchunov, D. Pustovoytov, Finite element modeling of shear strain in asymmetric and symmetric rolling in multi roll calibers, Procedia Engineering, 81 2469-2474, (2014)

13. A. Pesin, M. Chukin, A. Korchunov, D. Pustovoytov, Finite element modeling of shear strain in rolling with velocity asymmetry in multi-roll calibers, Key Engineering Materials. 622-623, 912-918, (2014)

14. B.B. Hundy, A.P. Green, A determination of plastic stress-strain relations, Journal of the Mechanics and Physics of Solids. 3, 16-21, (1954).

15. L.W. Meyer, S. Manwaring, Metallurgical Applications of Shock-Wave and High-Strain-Rate Phenomena, Marcel Dekker, New York, 1986.

16. J.R. Klepaczko, An experimental technique for shear testing at high and very high strain rates, International Journal of Impact Engineering. 46, 2539, (1994).

17. R.J. Clifton, R.W. Klopp, Pressure shear plate impact testing, in: Metals Handbook, Vol. 8, 9th edition, Mechanical Testing, ASM, Metals Park, Ohio, pp. 230-239, (1985).

18. J. Peirs, P. Verleysen, J. Degrieck, Novel technique for static and dynamic shear testing of Ti6Al4V sheet, Experimental Mechanics. 52, 729-741, (2011).

19. D. Rittel, S. Lee, G. Ravichandran, A shearcompression specimen for large strain testing, Experimental Mechanics. 42 58-64, (2002).

20. A. Dorogoy, D. Rittel, A. Godinger, Modification of the shear-compression specimen for large strain testing, Experimental Mechanics. 55, 1627-1639, (2015). 